\title{
Increased Oxidative Stress Response in Granulocytes from Older Patients with a Hip Fracture May Account for Slow Regeneration
}

\author{
Zhiyong Wang, ${ }^{1,2}$ Sabrina Ehnert, ${ }^{3}$ Christoph Ihle, ${ }^{3}$ Lilianna Schyschka, ${ }^{1}$ \\ Stefan Pscherer, ${ }^{4}$ Natascha C. Nussler, ${ }^{5}$ Karl F. Braun, ${ }^{1}$ Martijn Van Griensven, ${ }^{1}$ \\ Guobin Wang, ${ }^{2}$ Rainer Burgkart, ${ }^{6}$ Ulrich Stöckle, ${ }^{3}$ Florian Gebhard, ${ }^{7}$ \\ Helen Vester, ${ }^{1}$ and Andreas K. Nussler ${ }^{3}$ \\ ${ }^{1}$ Traumatology, Klinikum rechts der Isar, Technische Universität München, Ismaninger Straße 22, 81675 München, Germany \\ ${ }^{2}$ Department of Surgery, Union Hospital, Tongji Medical College, Huazhong University of Science and Technology, \\ Wuhan 430030, China \\ ${ }^{3}$ Siegfried Weller Institute for Trauma Research, BG Trauma Center Tübingen, Eberhard Karls University Tübingen, \\ Schnarrenbergstraße 95, 72076 Tübingen, Germany \\ ${ }^{4}$ Department of Diabetology, Klinikum Traunstein, Cuno-Niggl-Straße 3, 83278 Traunstein, Germany \\ ${ }^{5}$ Department of Surgery, Neuperlach Hospital, Städtisches Klinikum München GmbH, Oskar-Maria-Graf-Ring 51, \\ 81737 München, Germany \\ ${ }^{6}$ Clinic for Orthopedy, Klinikum rechts der Isar, Technische Universität München, Ismaninger Straße 22, 81675 München, Germany \\ ${ }^{7}$ Department of Trauma, Hand, Plastic and Reconstructive Surgery, Albert-Einstein University Ulm, Albert-Einstein-Allee 39, \\ $89081 \mathrm{Ulm}$, Germany
}

Correspondence should be addressed to Andreas K. Nussler; andreas.nuessler@gmail.com

Received 23 September 2013; Revised 23 January 2014; Accepted 23 January 2014; Published 2 March 2014

Academic Editor: Mohammad Abdollahi

Copyright (c) 2014 Zhiyong Wang et al. This is an open access article distributed under the Creative Commons Attribution License, which permits unrestricted use, distribution, and reproduction in any medium, provided the original work is properly cited.

Proximal femur fracture, a typical fracture of the elderly, is often associated with morbidity, reduced quality of life, impaired physical function and increased mortality. There exists evidence that responses of the hematopoietic microenvironment to fractures change with age. Therefore, we investigated oxidative stress markers and oxidative stress-related MAPK activation in granulocytes from the young and the elderly with and without fractured long bones. Lipid peroxidation levels were increased in the elderly controls and patients. Aged granulocytes were more sensitive towards oxidative stress induced damage than young granulocytes. This might be due to the basally increased expression of SOD-1 in the elderly, which was not further induced by fractures, as observed in young patients. This might be caused by an altered MAPK activation. In aged granulocytes basal p38 and JNK activities were increased and basal ERK1/2 activity was decreased. Following fracture, JNK activity decreased, while ERK1/2 and p38 activities increased in both age groups. Control experiments with HL60 cells revealed that the observed p38 activation depends strongly on age. Summarizing, we observed age-dependent changes in the oxidative stress response system of granulocytes after fractures, for example, altered MAPK activation and SOD-1 expression. This makes aged granulocytes vulnerable to the stress stimuli of the fracture and following surgery.

\section{Introduction}

Proximal femur fracture is a typical fracture of the elderly who frequently exhibit a lower bone mineral density, like, for example, osteopenia and osteoporosis, as well as muscle atrophy [1]. All over the world, approximately 2.6 million patients suffer from a hip fracture each year. Due to the anticipated demographic changes, this number is expected to be doubled in 40 years $[2,3]$. Despite the major progresses regarding surgical procedures, hip fractures are still a major public health concern. High postoperative complication rates negatively affect the general outcome as well as the quality 
TABLE 1: Information about donors.

\begin{tabular}{|c|c|c|c|c|}
\hline & \multicolumn{4}{|c|}{ Groups } \\
\hline & YH & YF & $\mathrm{OH}$ & OF \\
\hline Number of individuals & 22 & 12 & 23 & 19 \\
\hline Age range $(a)$ & 24 to 38 & 22 to 47 & 71 to 90 & 69 to 99 \\
\hline Age median (a) & 29.5 & 31 & 78 & 86 \\
\hline Number of male individuals & 11 & 9 & 9 & 7 \\
\hline Number of female individuals & 11 & 3 & 14 & 12 \\
\hline
\end{tabular}

YH: young controls; YF: young patients with a fracture of the long bones; OH: elderly controls; OF: elderly patients with a fracture of the long bones.

of life and cause high costs for the health care system $[2,4]$. However, little is known about the mechanisms influencing the recovery of the patients.

Changes in the microarchitecture and loss of bone substance may render the surgical treatment more complicated and delay fracture healing due to a disbalance of the function of osteoblasts and osteoclasts. However, these negative effects of bone remodeling do not provide sufficient explanation for the high rate of postoperative complications after hip fracture particularly in older patients [5]. The increased morbidity and mortality, for example, due to infections, in this subgroup of patients is more likely due to systemic changes which have negative effects on recovery [6]. Circulating cells of the immune system are directly exposed to the stress stimuli caused by the fracture and the following surgery. Thus, normally they should be able to respond to the external stress signal directly. In elderly patients, these responses are possibly impaired due to alterations of the immune system.

Granulocytes and monocyte derived macrophages constitute the first lines of defense against bacterial infections. After a trauma, neutrophils migrate to the site of injury within minutes, where mitogen-activated protein kinase (MAPK) signaling occurs in granulocytes as a concomitant circumstance to extracellular stimuli that affect the functions of the cell, like, for example, proliferation, differentiation, or apoptosis $[7,8]$.

MAPKs that have been reported to be involved in these processes are JNK, ERK1/2, and p38 [9-16]. These MAPKs are all directly affected by oxidative stress stimuli $[7,9]$. Their activation may induce cellular defense mechanisms, for example, the expression of the antioxidative enzyme superoxide dismutase (SOD-1) which directly eliminates reactive oxygen species (ROS) by catalyzing the dismutation of superoxide to hydrogen peroxide and molecular oxygen [17].

Thus, the aim of the present study consisted in investigating oxidative stress levels, oxidative stress induced toxicity, and the related MAPK activation in granulocytes from young ( $<50$ years of age) and elderly ( $>65$ years of age) patients with fractures of the long bones and from healthy controls. This comparison should enable us to draw conclusions about alterations of the response of granulocytes to the stress stimuli of a fracture and following surgery in young and old patients.

\section{Materials and Methods}

Phosphate buffered saline, fetal calf serum, and RPMI1640 were purchased from PAA Laboratories GmbH (Pasching,
Austria). Complete protease inhibitor was obtained from Roche (Mannheim, Germany). All other chemicals were purchased from Sigma (Munich, Germany).

2.1. Ethics Statement. Blood sampling was conducted in accordance with the Declaration of Helsinki (1964) and its amendments. The study protocol was approved by the hospital's Ethics Committee and informed consent was obtained from all subjects. Patients with chronic diseases were not included in the study.

2.2. Sample Population. $45 \mathrm{~mL}$ venous blood (36 mL EDTA (ethylenediaminetetraacetic acid) blood and $9 \mathrm{~mL}$ serum blood) was collected from each patient. Two different age groups were studied: young patients aged less than 50 years and elderly patients aged over 65 years. In both age groups, we distinguished between healthy controls and patients with a fracture of the long bones. The study population consisted of 22 young healthy controls (YH), 23 old healthy controls $(\mathrm{OH}), 12$ young patients (YF), and 19 old patients (OF). In both groups of patients, the blood sampling was performed within $6 \mathrm{~h}$ after the operative reposition of the fracture of one of the long bones of the lower extremity. Demographic data is summarized in Table 1.

2.3. Granulocyte Isolation. The granulocytes were isolated by MACS (magnetic cell separation) using CD15 magnetic microbeads according to the manufacturer's instructions (Miltenyi Biotec, Cologne, Germany) or by density gradient centrifugation as described in [18]. Immediately after their isolation, the granulocytes were lysed for Western blot analysis. Cell purity was over $95 \%$ as assessed by flow cytometry.

2.4. HL60 Cell Culture. The HL60 granulocyte cells were expanded in RMPI 1640 medium containing 10\% fetal calf serum, $2 \mathrm{mM}$ L-glutamine, $100 \mathrm{U} / \mathrm{mL}$ penicillin, and $100 \mu \mathrm{g} / \mathrm{mL}$ streptomycin at $37^{\circ} \mathrm{C}$ and $5 \% \mathrm{CO}_{2}$. For maturation culture medium was supplemented with $1 \%$ dimethylsulfoxide. Prior to the experiment, the cells were serum-starved overnight. After $1 \mathrm{~h}$ stimulation with culture medium containing 10\% sera from young and old patients and controls, cells were lysed for Western blot analysis.

2.5. Quantification of Lipid Peroxidation to Assess Oxidative Stress Levels. In order to measure the oxidative stress, lipid peroxidation was determined through the malondialdehyde 


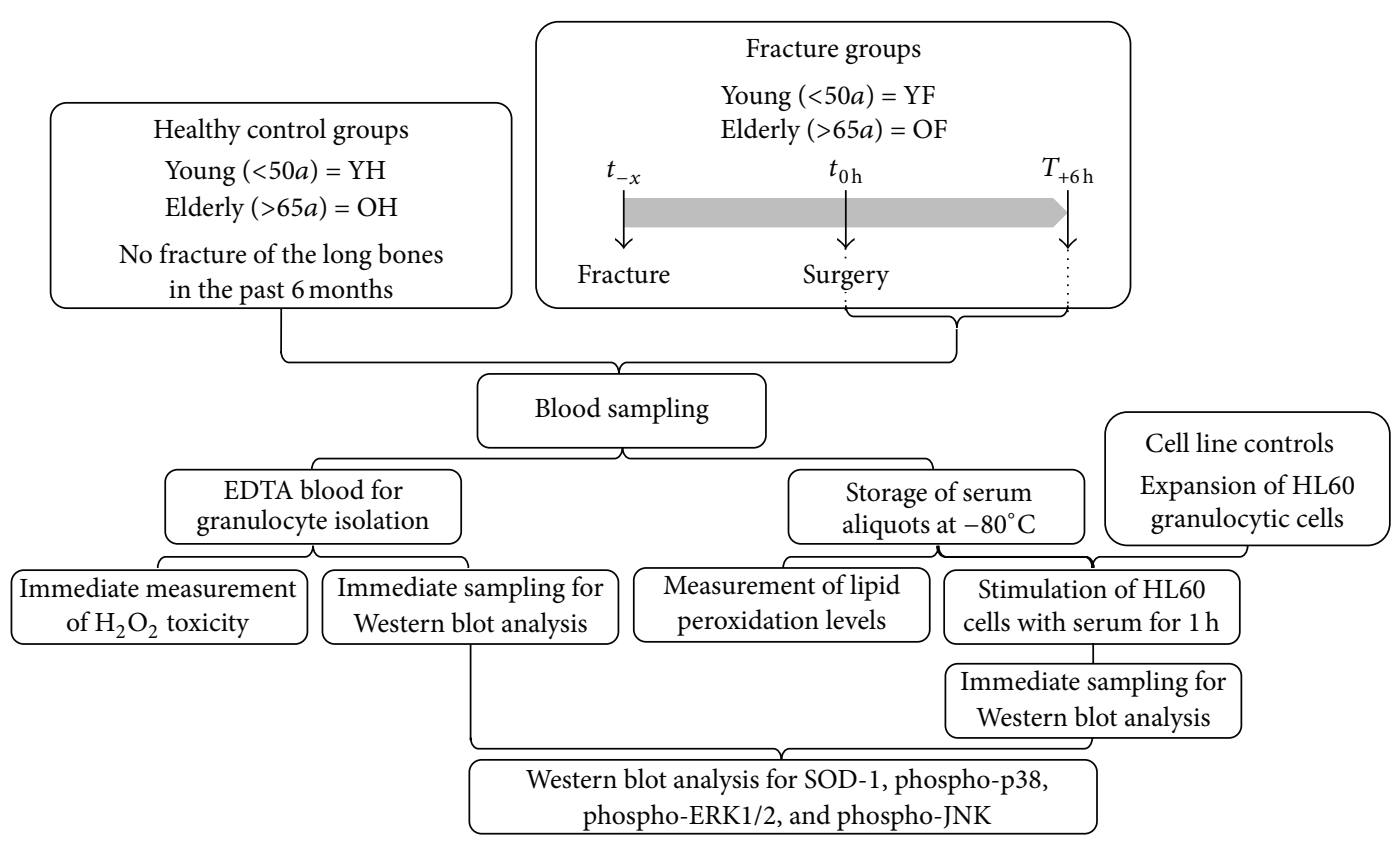

Figure 1: Overview on the experimental setup.

assay. Briefly, $15 \mu \mathrm{L}$ serum was incubated with $45 \mu \mathrm{L}$ thiobarbituric acid solution $(0.33 \%$ thiobarbituric acid in $11.1 \%$ acetic acid) for $1 \mathrm{~h}$ at $100^{\circ} \mathrm{C}$. The samples were diluted with distilled water and their fluorescence was measured immediately at an excitation/emission wavelength of 544/590 nm. Known concentrations of 1,1,3,3-tetraethoxypropane were used as a standard control.

2.6. Viability Measurement. The cells' viability was determined by resazurin conversion. Briefly, cells $\left(\sim 7 * 10^{5}\right.$ cells/well) were stimulated with various concentrations of $\mathrm{H}_{2} \mathrm{O}_{2}(0,0.002,0.004,0.008,0.016,0.03,0.06,0.125,0.25,0.5$, and $1 \%) .1 / 10$ volume of a $0.025 \%(\mathrm{w} / \mathrm{v})$ resazurin solution (in phosphate buffered saline) was added to the cells. After $1 \mathrm{~h}$ incubation at $37^{\circ} \mathrm{C}$ fluorescence was measured $(\mathrm{ex} / \mathrm{em}=$ $544 / 590 \mathrm{~nm}$ ) and corrected to background control (no cells). Viability is given as \% of untreated control.

2.7. Western Blot Analysis. Cells were lysed in freshly prepared ice-cold RIPA buffer (50 $\mathrm{mM}$ tris(hydroxymethyl)aminomethan, $250 \mathrm{mM} \mathrm{NaCl}, 2 \%$ Nonidet-P40, $2.5 \mathrm{mM}$ EDTA, $0.1 \%$ sodium dodecyl sulfate (SDS), $0.5 \%$ deoxycholic acid, protease and phosphatase inhibitors, $\mathrm{pH}=$ 7.2). Protein concentration was determined by micro-Lowry [19]. 30 to $50 \mu \mathrm{g}$ total protein was separated by SDS-PAGE and transferred to nitrocellulose membranes (Roth, Karlsruhe, Germany). Unspecific binding sites were blocked with $5 \%$ bovine serum albumin in TBST solution $(25 \mathrm{mM}$ tris(hydroxymethyl)-aminomethan, $137 \mathrm{mM} \mathrm{NaCl}, 2.7 \mathrm{mM}$ $\mathrm{KCl}, 0.05 \%$ Tween-20, $\mathrm{pH}=7.4$ ) for $1 \mathrm{~h}$ at RT. After overnight incubation with primary antibodies (Cell Signaling, Beverly, USA) diluted $1: 1,000$ in TBST at $4^{\circ} \mathrm{C}$, membranes were incubated with the corresponding horseradish peroxidaselabeled secondary antibodies (1:10,000 in TBST) for $2 \mathrm{~h}$ at RT.
Chemiluminescent signals were detected with X-ray films or a charge-coupled device camera (CCD camera). In order to compare specific signals on different blots, a uniform positive control (HEK293T cells) was included for each membrane. Signal intensities were determined by densitometry using the Image software (NIH, Bethesda, USA) and normalized to the corresponding GAPDH signals.

2.8. Statistics. A flow diagram on the experimental setup is given in Figure 1. Results are expressed as mean \pm SEM. The amount of donors $(N)$ and replicates $(n)$ is given separately for each experiment. Datasets were compared by one-way analysis of variance or by the Student's $t$-test (GraphPad Prism Software, LaJolla, CA, USA). $P<0.05$ was taken as the minimum level of significance.

\section{Results}

3.1. The Elderly Show Increased Lipid Peroxidation Levels Representing Elevated Oxidative Stress Levels. In order to determine the levels of oxidative stress in the donors, the lipid peroxidation in the serum $(N=10$ /group, $n=2)$ was measured. The levels of basal lipid peroxidation in the older controls were approximately twice as high as those of young controls $(P<0.001)$. Interestingly, after the occurrence of a fracture a significant increase of about $25 \%$ of the lipid peroxidation was detected only in the older patients. Young patients with a fracture of the long bone showed no statistic increase in lipid peroxidation (Figure 2(a)).

3.2. Viability Measurement Indicates That Aged Granulocytes Are More Sensitive Towards $\mathrm{H}_{2} \mathrm{O}_{2}$ Induced Toxicity as Compared to Young Granulocytes. In order to investigate whether the increased oxidative stress affects the viability of 


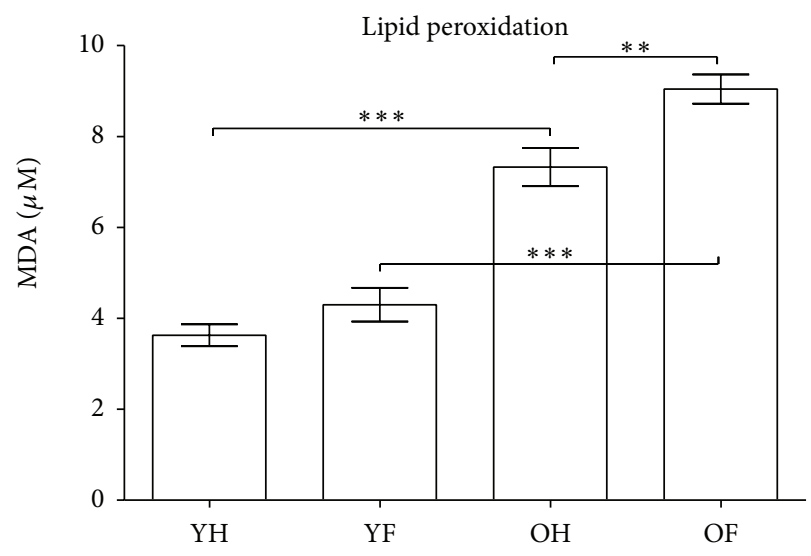

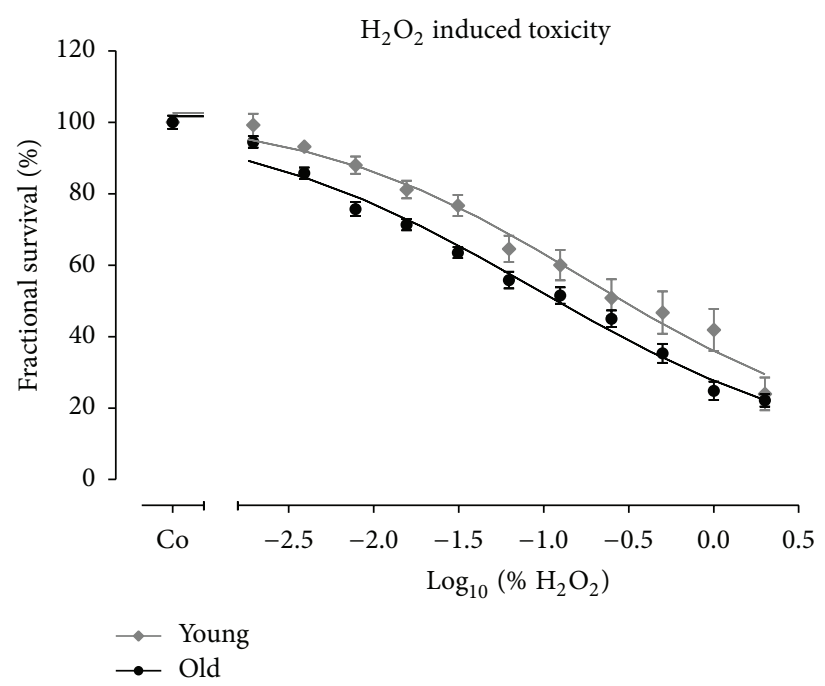

(b)

Figure 2: Aged granulocytes are more sensitive towards $\mathrm{H}_{2} \mathrm{O}_{2}$ induced toxicity as young granulocytes. (a) Levels of oxidative stress were determined by measuring lipid peroxidation in sera of young controls $(\mathrm{YH})$, young patients with a fracture of the long bones (YF), elderly controls $(\mathrm{OH})$, and elderly patients with a fracture of the long bones $(\mathrm{OF}) . N=10$ and $n=2$ per group. ${ }^{* *} P>0.01$ and ${ }^{* * *} P<0.001$ as determined by one-way ANOVA. (b) Granulocytes from young controls $(N=5, n=4)$ and elderly controls $(N=5, n=4)$ were stimulated with various concentrations of $\mathrm{H}_{2} \mathrm{O}_{2}(0,0.002,0.004,0.008,0.016,0.03,0.06,0.125,0.25,0.5$, and $1 \%)$. After $1 \mathrm{~h}$ viability was assessed by resazurin conversion. $\mathrm{EC}_{50 / \text { young }}=0.18 \%$ and $\mathrm{EC}_{50 / \text { old }}=0.09 \%(P<0.05$ as determined by Student's $t$-test $)$.

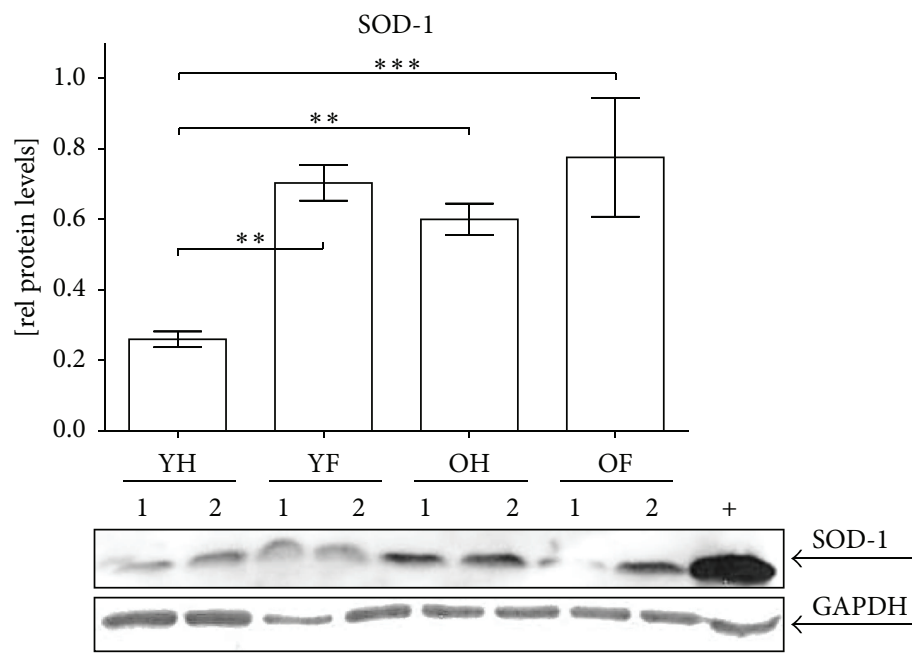

FIGURE 3: Altered SOD-1 expression in aged granulocytes. Representative Western blot for SOD-1. GAPDH was used as a loading control and for normalization. For intermembrane comparison HEK293T cells $(+)$ were used. Densitometric analysis of all donors (YH: $N=22 / \mathrm{YF}$ : $N=12 / \mathrm{OH}: N=23 / \mathrm{OF}: N=19)$ was performed to determine the relative expression levels of SOD-1. ${ }^{* *} P>0.01$ and ${ }^{* * *} P<0.001$ as determined by one-way ANOVA.

granulocytes, we stimulated granulocytes from young and elderly controls with various concentrations of $\mathrm{H}_{2} \mathrm{O}_{2}(0$, $0.002,0.004,0.008,0.016,0.03,0.06,0.125,0.25,0.5$, and $1 \%)$. After $1 \mathrm{~h}$ viability was assessed by resazurin conversion. Granulocytes from elderly controls $(N=5, n=4)$ were more sensitive towards $\mathrm{H}_{2} \mathrm{O}_{2}$ induced toxicity than granulocytes from young controls $(N=5, n=4)$, represented by an $\mathrm{EC}_{50}$ of $0.09 \%$ and $0.18 \%$, respectively $(P<0.05)$ (Figure $2(b)$ ).
3.3. Altered SOD-1 Expression in Aged Granulocytes Is Detected by Western Blot Analysis. In order to investigate why oxidative stress strongly affects aged granulocytes, we investigated the expression levels of SOD-1 by Western blot (Figure 3). Densitometric analysis revealed that the basal expression levels of SOD-1 were significantly elevated (2.2-fold) in the older controls in comparison to the young controls. After trauma and following surgery, the SOD-1 levels significantly 


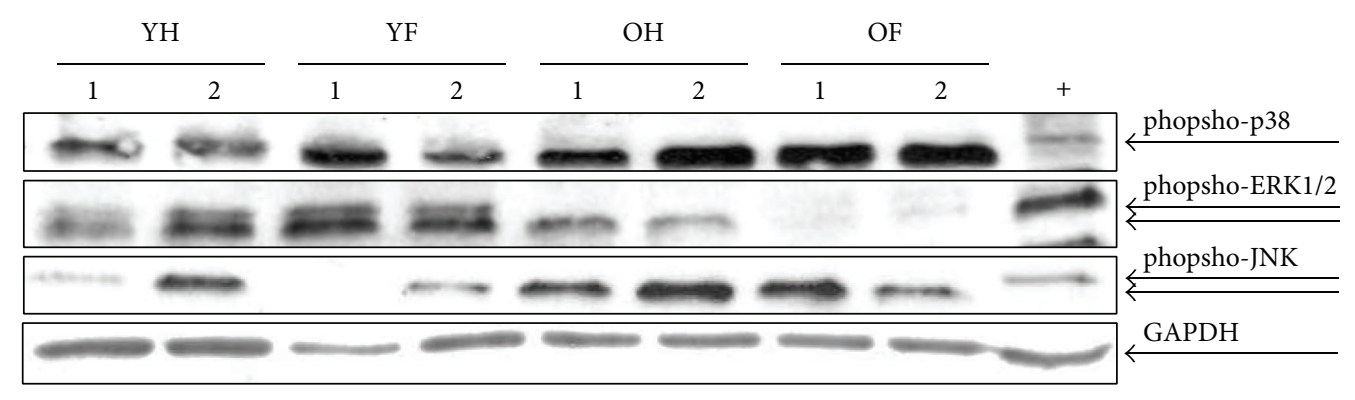

(a)

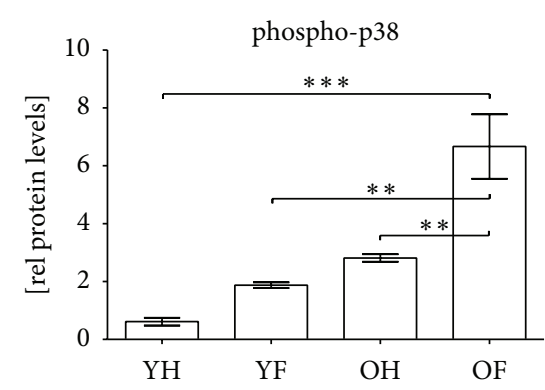

(b)

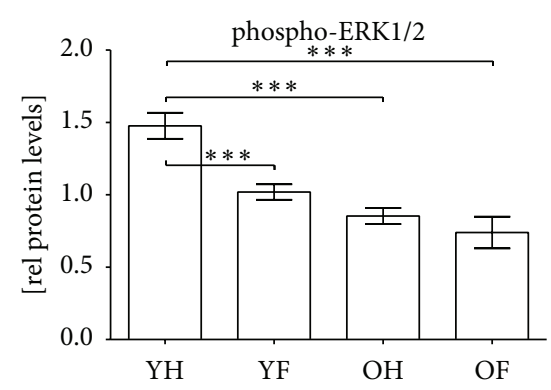

(c)

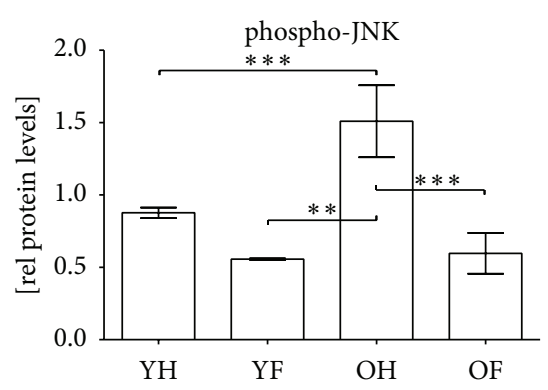

(d)

FIGURE 4: Altered activation of MAPKs in granulocytes of elderly patients and controls. (a) Representative Western blot for phospho-p38, phospho-ERK1/2, and phospho-JNK in granulocytes of young controls (YH), young patients with a fracture of the long bones (YF), elderly controls $(\mathrm{OH})$, and elderly patients with a fracture of the long bones (OF). GAPDH was used as a loading control and for normalization. For intermembrane comparison HEK293T cells $(+)$ were used. Densitometric analysis of all donors $(\mathrm{YH}: N=22 / \mathrm{YF}: N=12 / \mathrm{OH}: N=23 / \mathrm{OF}$ : $N=19$ ) was performed to determine the relative expression levels of (b) phospho-p38, (c) phospho-ERK1/2, and (d) phospho-JNK. ${ }^{* *} P>$ 0.01 and ${ }^{* * *} P<0.001$ as determined by one-way ANOVA.

increased in the young patients (2.7-fold). Interestingly, in older patients, the basally elevated expression levels of the SOD-1 did not further increase after trauma and following surgery.

\subsection{Altered MAPKs Activation in Granulocytes of the Elderly} Is Detected by Western Blot Analysis. In order to investigate whether the increased oxidative stress may affect the stress response of granulocytes, we investigated activation (phosphorylation) of the MAPKs p38, ERK1/2, and JNK by Western blot (Figure 4(a)). Basal activation of p38 was increased 4.5fold in elderly controls as compared to young controls. After the occurrence of a fracture, a significant increase (2.5-fold) in the p38 activation was observed only in elderly patients. In young patients, however, the increase in p38 activity was not significant and did not reach the basal levels of older controls (Figure 4(b)). On the contrary, basal activation of ERK1/2 signaling was significantly decreased (about 40\%) in elderly controls. After a fracture, ERK1/2 activation was further decreased only in young patients by about $30 \%$ (Figure 4(c)). Similar to p38 activation, basal JNK activation was significantly increased (1.9-fold) in elderly controls in comparison to young controls. After a fracture of the long bones, phospho-JNK levels decreased in both groups with the decrease being more pronounced in the group of the elderly (Figure 4(d)). Serum Stimulated HL60 Cells in Comparison to Granulocytes.
In order to analyze whether the activation of MAPKs that has been observed in granulocytes is age-dependent or caused by the observed changes in the cellular microenvironment (serum), the activation of the three MAPKs in matured HL60 cells (considered to have the same age) cultured in the presence of the patients' sera was investigated (Figure 5(a)). Contrary to the granulocytes, basal activation of p38 was decreased (about 30\%) in the HL60 cells stimulated with sera of the elderly controls in comparison to the HL60 cells stimulated with sera of the young controls. HL60 cells stimulated with patients' sera showed a significant decrease (2.5-fold) in p38 activation only in the young group (Figure 5(b)). Similar to the granulocytes, basal activation of ERK1/2 signaling was significantly decreased, by about $60 \%$, in HL60 cells stimulated with sera of the elderly controls. In both age groups activation of ERK1/2 was reduced in HL60 cells stimulated with patients' sera as compared to stimulation with control sera (Figure 5(c)). On the contrary to granulocytes, basal JNK activation was significantly decreased, by 50\%, in HL60 cells stimulated with sera of the elderly controls as compared to stimulation with control sera. The phospho-JNK levels further decreased in HL60 cells stimulated with patients' sera, with the decrease being more pronounced in the young group (Figure 5(d)).

\section{Discussion}

Hip fracture is a typical fracture of the elderly and its risk directly correlates with bone mineral density and bone 


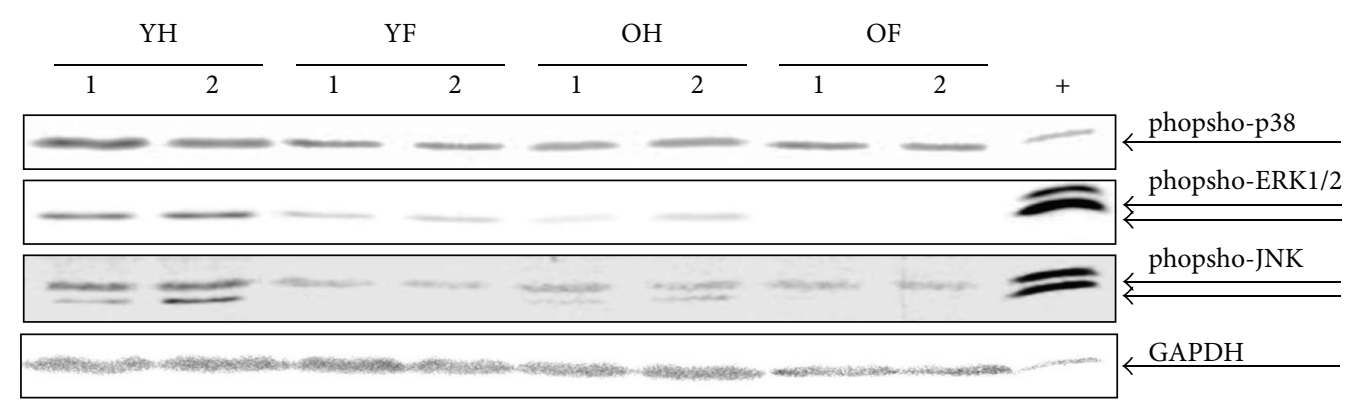

(a)

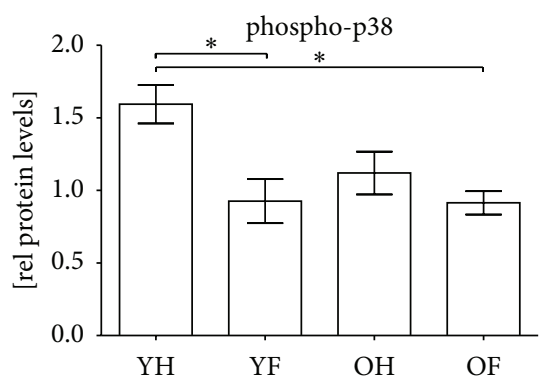

(b)

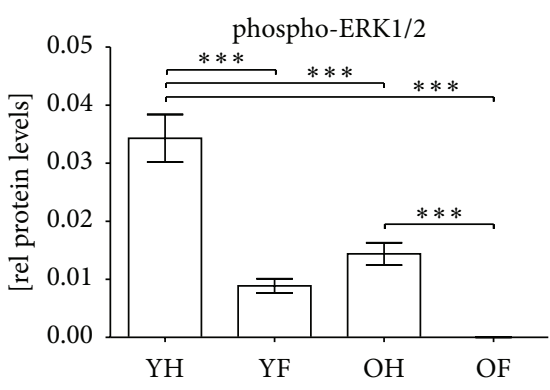

(c)

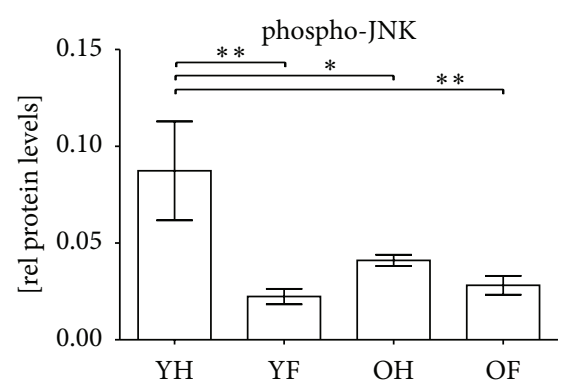

(d)

FIGURE 5: Inverted p38 activation in serum-conditioned HL60 cells in comparison to granulocytes. (a) Representative Western blot for phospho-p38, phospho-ERK1/2, and phospho-JNK in HL60 cells conditioned with serum of young controls (YH), young patients with a fracture of the long bones (YF), elderly controls (OH), and elderly patients with a fracture of the long bones (OF). GAPDH was used as a loading control and for normalization. For intermembrane comparison HEK293T cells (+) were used. Densitometric analysis of all samples ( $N=10, n=2$ /group) was performed to determine relative expression levels of (b) phospho-p38, (c) phospho-ERK1/2, and (d) phospho-JNK. ${ }^{*} P>0.05,{ }^{* *} P>0.01$ and ${ }^{* * *} P<0.001$ as determined by one-way ANOVA.

strength $[20,21]$. Thus, facing the current demographic changes with an increasing number of older people, the treatment of hip fractures represents an important problem for the public health systems [3,4]. Despite all surgical advances over the past years, hip fractures are still accompanied by high complication rates, and therefore by an increased disability, morbidity, and mortality. While onethird of the patients require a higher level of long-term care, the in-hospital mortality accounts to almost $10 \%$ and the one-year mortality accounts for around $27 \%$ [22]. This high complication rate may be due to alterations in the immune system in the elderly, which render these patients prone to infections. Granulocytes are among the first lines of defense against bacterial infections. They are circulating immune cells and therefore they are directly exposed to the stress stimuli generated by the fracture and by the operative fracture fixation. Therefore, the aim of this project consisted in investigating the stress response of granulocytes obtained from young ( $<50$ years of age) and elderly ( $>65$ years of age) patients with a fracture of one of the long bones. Healthy individuals of both age groups served as control groups.

In the serum of older controls an increase in oxidative stress, represented by increased lipid peroxidation levels, was observed. ROS, particularly superoxide and its derivatives, induce an accumulation of oxidative damage to macromolecules in the cell, including proteins, lipids, and DNA, which in turn causes aging and eventually cell death [23, 24].
After the occurrence of a fracture and the following surgical reposition of the bone, the lipid peroxidation levels increased in the elderly group. Interestingly, in young patients, the levels of oxidative stress did not significantly increase after the occurrence of a fracture. This observation suggests that the young patients are able to actively reduce excessive amounts of ROS and reactive oxygen and nitrogen intermediated (ROI and RNI), for example, by expressing antioxidative enzymes. This is supported by our finding that aged granulocytes were more sensitive towards $\mathrm{H}_{2} \mathrm{O}_{2}$ induced cellular damage than granulocytes from young controls. SOD-1 is a free radical scavenger which is believed to act as the first line of defense against oxidative damage by catalyzing the conversion of superoxide radicals to hydrogen peroxide, which can then be reduced to water $[25,26]$. In granulocytes of elderly controls, we observed increased basal SOD-1 levels. The increased basal SOD-1 levels are probably at maximum and cannot be further elevated after fractures. After the occurrence of a fracture, SOD-1 levels significantly increased only in the young patients. These findings confirm the oxidative stress theory of aging by Harman, in which he predicts that the imbalance between the formation of oxidative stress and antioxidant defense mechanisms results in a steady accumulation of oxidative damage in a variety of macromolecules [27].

The increase in oxidative stress in turn affects circulating immune cells that are directly exposed to the stimuli. 
Granulocytes migrate to the site of injury within minutes. In particular, the MAPKs JNK, p38, and ERK1/2 are directly affected by oxidative stress stimuli in these cells [7, 9].

Basal p38 signaling was increased in the granulocytes of the older controls. After the occurrence of a fracture, a considerable induction of p38 signaling was observed only in the older patients. After its activation, p38 initiates a signaling cascade that regulates the synthesis of a variety of proinflammatory mediators, like tumor necrosis factor $\alpha$ (TNF- $\alpha$ ) [28], which was increased in the serum of older patients and controls. The basal JNK signaling was elevated in the elderly controls in a similar fashion to that of the p38 signaling. However, after the occurrence of a fracture, the activation of JNK dropped in young and older patients. Acute and transient activation of JNK is reported to induce cell proliferation and survival, while a prolonged and sustained activation of JNK may promote cell apoptosis [29] which is triggered by TNF- $\alpha$ and ROS [30] that are both elevated in the elderly patients and controls. Contrary to the p38 and JNK signaling, the basal ERK1/2 signaling was significantly lower in the elderly than in the young controls. After the occurrence of a fracture, ERK1/2 signaling was significantly lower in the young patients than in the young controls. However, in the elderly, this difference between the patients and the controls in the ERK1/2 signaling was much smaller. These findings are supported by the work of Schieven, in which it is shown that active p38 signaling may inhibit ERK1/2 signaling [28]. This is especially important as Larbi and coworkers were able to prove that the functional ERK1/2 signaling was able to inhibit granulocyte apoptosis which had been induced by Granulocyte-macrophage colony-stimulating factor (GMCSF) [31]. Thus, granulocytes of older people have a higher susceptibility to apoptosis in the presence of elevated GMCSF levels due to their impaired ERK1/2 signaling. Due to their increased rate of apoptosis in their granulocytes, elderly patients are more sensitive to infections following a trauma than young patients.

In order to investigate whether the observed alterations in the MAPK signaling are age-dependent or due to the observed changes in the microenvironment (cytokines, chemokines, and oxidative stress stimuli), we stimulated HL60 granulocytes (considered to have the same age) with patients' sera and investigated the activation of the MAPKs. ERK1/2 and JNK signaling were decreased in the elderly patients and controls and further decreased after a fracture analogously to the number of controls. However, contrary to the number of granulocytes, basal p38 signaling was decreased in the elderly controls and even further decreased after fracture. This observation suggests that p38 signaling is particularly prone to age-related changes. The finding of elevated p38 signaling in the granulocytes of the elderly patients is especially interesting as recent studies have demonstrated that it plays a key role in the persistent pain sensitization via neuronal and glial mechanisms [32-34]. Pain relief, before and after surgery, is one of the first priorities for patients with hip fractures, as adequate pain control may reduce complications, like, for example, cardiovascular events and restoring ambulation [6].

\section{Summary}

In summary, our data show an increase in oxidative stress levels in the elderly patients and controls. The strong upregulation of SOD-1 in granulocytes after fracture suggests that young patients are able to actively reduce ROS, ROI, and RNI, whereas in elderly patients, SOD-1 expression seems to have reached its limits already in a normal state. This might explain that aged granulocytes react more sensitively towards oxidative stress induced damage than the young granulocytes. The activation of MAPKs in the granulocytes indicates that young and old granulocytes react differently to the oxidative stress stimuli. p38 is especially age dependent. The shift in ERK1/2 and JNK signaling may sensitize the granulocytes of the elderly towards damage caused by oxidative stress stimuli.

\section{Conclusion}

Our data suggest that a balanced antioxidative response in granulocytes of elderly patients might protect them from additional stress stimuli, for example, fracture and following operation. A flavonoid rich diet is reported to induce the expression of antioxidative enzymes in various cell types [3538]. However, this induction of the expression of antioxidative enzymes can only be reached, when flavonoids are consumed at high doses over a long period of time. This might even improve bone quality and thus reduce fracture risk, as the rat study with retinoic acid-induced bone loss from Orsolic and coworkers suggests [38]. However, in patients suffering from a fracture the first stress stimuli already occurred. Therefore, the clinic treatment can only be preventive against the second stress stimuli from the following operation, for example, by using drugs with strong antioxidative properties that can be applied in defined doses [35]. There have been first positive reports applying this strategy to prevent organ damage after abdominal surgery $[39,40]$. However, if this treatment may also protect the immune cells has to be further evaluated in a controlled clinical trial.

\section{Abbreviations}

ERK1/2: Extracellular signal-regulated kinases 1 and 2

GM-CSF: Granulocyte-macrophage colony-stimulating factor

JNK: c-Jun N-terminal kinase

MACS: Magnetic cell separation

MAPK: Mitogen-activated protein kinase

RNI: Reactive nitrogen intermediates

ROI: Reactive oxygen intermediates

ROS: $\quad$ Reactive oxygen species

SOD-1: $\quad$ Superoxide dismutase 1

TNF- $\alpha$ : Tumor necrosis factor $\alpha$.

\section{Conflict of Interests}

The authors declare that there is no conflict of interests regarding the publication of this paper. 


\section{Authors' Contribution}

Zhiyong Wang, Sabrina Ehnert, Florian Gebhard, Helen Vester, and Andreas K. Nussler contributed equally to this paper

\section{Acknowledgments}

We would like to thank Angela Wottge and Patrysya Lacorte for their excellent technical assistance. The present work was supported by the Fresenius Foundation (Project no. A116). Zhiyong Wang was supported by the Chinese Scholarship Council and the B. Braun Foundation.

\section{References}

[1] C. D. Rubin, "Evaluation and management of hip fracture risk in the aged," American Journal of the Medical Sciences, vol. 343, no. 3, pp. 233-242, 2012.

[2] C. A. Brauer, M. Coca-Perraillon, D. M. Cutler, and A. B. Rosen, "Incidence and mortality of hip fractures in the United States," Journal of the American Medical Association, vol. 302, no. 14, pp. 1573-1579, 2009.

[3] A. Icks, B. Haastert, G. Glaeske, U. Stumpf, J. Windolf, and F. Hoffmann, "Correction factor for the analysis of the hip fracture incidence-differences between age, sex, region, and calendar year," Wiener klinische Wochenschrift, vol. 124, no. 11-12, p. 391, 2012.

[4] A. P. Diamantopoulos, G. Rohde, I. Johnsrud et al., "Incidence rates of fragility hip fracture in middle-aged and elderly men and women in Southern Norway," Age and Ageing, vol. 41, no. 1, Article ID afr114, pp. 86-92, 2012.

[5] K. S. Hapuarachchi, R. S. Ahluwalia, and M. G. Bowditch, "Neck of femur fractures in the over 90s: a select group of patients who require prompt surgical intervention for optimal results," Journal of Orthopaedics and Traumatology. In press.

[6] N. T. O’Malley, M. Blauth, N. Suhm, and S. L. Kates, "Hip fracture management, before and beyond surgery and medication: a synthesis of the evidence," Archives of orthopaedic and trauma surgery, vol. 131, no. 11, pp. 1519-1527, 2011.

[7] H. Gehart, S. Kumpf, A. Ittner, and R. Ricci, "MAPK signalling in cellular metabolism: stress or wellness?" EMBO Reports, vol. 11, no. 11, pp. 834-840, 2010.

[8] G. Pearson, F. Robinson, T. B. Gibson et al., "Mitogen-activated protein (MAP) kinase pathways: regulation and physiological functions," Endocrine Reviews, vol. 22, no. 2, pp. 153-183, 2001.

[9] P. Cohen, "Targeting protein kinases for the development of anti-inflammatory drugs," Current Opinion in Cell Biology, vol. 21, no. 2, pp. 317-324, 2009.

[10] A. Cuenda and S. Rousseau, "p38 MAP-Kinases pathway regulation, function and role in human diseases," Biochimica et Biophysica Acta, vol. 1773, no. 8, pp. 1358-1375, 2007.

[11] D. N. Dhanasekaran and E. P. Reddy, "JNK signaling in apoptosis," Oncogene, vol. 27, no. 48, pp. 6245-6251, 2008.

[12] S. Kumar, J. Boehm, and J. C. Lee, "P38 MAP kinases: key signalling molecules as therapeutic targets for inflammatory diseases," Nature Reviews Drug Discovery, vol. 2, no. 9, pp. 717726, 2003.

[13] J. Liu and A. Lin, "Role of JNK activation in apoptosis: a doubleedged sword," Cell Research, vol. 15, no. 1, pp. 36-42, 2005.
[14] M. Loesch and G. Chen, “The p38 MAPK stress pathway as a tumor suppressor or more?" Frontiers in Bioscience, vol. 13, no. 9, pp. 3581-3593, 2008.

[15] A. Y. Ring, K. M. Sours, T. Lee, and N. G. Ahn, "Distinct patterns of activation-dependent changes in conformational mobility between ERK1 and ERK2," International Journal of Mass Spectrometry, vol. 302, no. 1-3, pp. 101-109, 2011.

[16] T. M. Thornton and M. Rincon, "Non-classical p38 map kinase functions: cell cycle checkpoints and survival," International Journal of Biological Sciences, vol. 5, no. 1, pp. 44-52, 2009.

[17] J. D. Crapo, T. Oury, C. Rabouille, J. W. Slot, and L.-Y. Chang, "Copper,zinc superoxide dismutase is primarily a cytosolic protein in human cells," Proceedings of the National Academy of Sciences of the United States of America, vol. 89, no. 21, pp. 10405-10409, 1992.

[18] L. V. Jepsen and T. Skottun, "A rapid one-step method for the isolation of human granulocytes from whole blood," Scandinavian Journal of Clinical and Laboratory Investigation, vol. 42, no. 3, pp. 235-238, 1982.

[19] O. H. Lowry, N. J. Rosebrough, A. L. Farr, and R. J. Randall, "Protein measurement with the Folin phenol reagent," The Journal of biological chemistry, vol. 193, no. 1, pp. 265-275, 1951.

[20] M. Fernández-Ruiz, J. M. Guerra-Vales, R. Trincado, M. J. Medrano, J. Benito-León, and F. Bermejo-Pareja, "Hip fracture in three elderly populations of central Spain: data from the NEDICES study," Internal and Emergency Medicine, pp. 1-9, 2011.

[21] J. L. Hernandez, J. M. Olmos, M. A. Alonso et al., "Trend in hip fracture epidemiology over a 14-year period in a Spanish population," Osteoporosis International, vol. 17, no. 3, p. 464, 2006.

[22] S. Karaca, E. Ayhan, H. Kesmezacar, and O. Uysal, "Hip fracture mortality: is it affected by anesthesia techniques?" Anesthesiology Research and Practice, vol. 2012, Article ID 708754, 5 pages, 2012.

[23] K. B. Beckman and B. N. Ames, "The free radical theory of aging matures," Physiological Reviews, vol. 78, no. 2, pp. 547-581, 1998.

[24] A. B. Salmon, A. Richardson, and V. I. Pérez, "Update on the oxidative stress theory of aging: does oxidative stress play a role in aging or healthy aging?" Free Radical Biology and Medicine, vol. 48, no. 5, pp. 642-655, 2010.

[25] F. L. Muller, W. Song, Y. Liu et al., "Absence of CuZn superoxide dismutase leads to elevated oxidative stress and acceleration of age-dependent skeletal muscle atrophy," Free Radical Biology and Medicine, vol. 40, no. 11, pp. 1993-2004, 2006.

[26] R. Noor, S. Mittal, and J. Iqbal, "Superoxide dismutaseapplications and relevance to human diseases," Medical Science Monitor, vol. 8, no. 9, pp. RA210-RA215, 2002.

[27] D. Harman, "Aging: a theory based on free radical and radiation chemistry," Journal of gerontology, vol. 11, no. 3, pp. 298-300, 1956.

[28] G. L. Schieven, "The p38 $\alpha$ kinase plays a central role in inflammation," Current Topics in Medicinal Chemistry, vol. 9, no. 11, pp. 1038-1048, 2009.

[29] J.-J. Ventura, A. Hübner, C. Zhang, R. A. Flavell, K. M. Shokat, and R. J. Davis, "Chemical genetic analysis of the time course of signal transduction by JNK," Molecular Cell, vol. 21, no. 5, pp. 701-710, 2006.

[30] S. Papa, C. Bubici, F. Zazzeroni et al., "The NF- $\kappa$ B-mediated control of the JNK cascade in the antagonism of programmed cell death in health and disease," Cell Death and Differentiation, vol. 13, no. 5, pp. 712-729, 2006. 
[31] A. Larbi, N. Douziech, C. Fortin, A. Linteau, G. Dupuis, and T. Fulop Jr., "The role of the MAPK pathway alterations in GM-CSF modulated human neutrophil apoptosis with aging," Immunity and Ageing, vol. 2, article 6, 2005.

[32] R.-R. Ji and G. Strichartz, "Cell signaling and the genesis of neuropathic pain," Science's STKE, vol. 2004, no. 252, p. reE14, 2004.

[33] S.-X. Jin, Z.-Y. Zhuang, C. J. Woolf, and R.-R. Ji, "p38 mitogenactivated protein kinase is activated after a spinal nerve ligation in spinal cord microglia and dorsal root ganglion neurons and contributes to the generation of neuropathic pain," Journal of Neuroscience, vol. 23, no. 10, pp. 4017-4022, 2003.

[34] K. Obata, H. Yamanaka, K. Kobayashi et al., "Role of mitogenactivated protein kinase activation in injured and intact primary afferent neurons for mechanical and heat hypersensitivity after spinal nerve ligation," Journal of Neuroscience, vol. 24, no. 45, pp. 10211-10222, 2004.

[35] S. Ehnert, S. Döbele, K. F. Braun et al., "N-Acetylcyteine and flavonoid rich diet: the protective effect of 15 different antioxidants on cigarette smoke-damaged primary human osteoblasts," Advances in Bioscience and Biotechnology, vol. 3, p. 1129, 2012.

[36] B. Kling, D. Bucherl, P. Palatzky et al., "Flavonoids, flavonoid metabolites, and phenolic acids inhibit oxidative stress in the neuronal cell line HT-22 monitored by ECIS and MTT assay: a comparative study," Journal of Natural Products. In press.

[37] S. Liu, W. Hou, P. Yao et al., "Heme oxygenase-1 mediates the protective role of quercetin against ethanol-induced rat hepatocytes oxidative damage," Toxicology in Vitro, vol. 26, no. 1, pp. 74-80, 2012.

[38] N. Orsolic, E. Goluza, D. Dikic et al., "Roleof flavonoids on oxidative stress and mineral contents in the retinoic acidinduced bone loss model of rat," European Journal of Nutrition. In press.

[39] F. D’Amico, A. Vitale, D. Piovan et al., "Use of N-acetylcysteine during liver procurement: a prospective randomized controlled study," Liver Transplantation, vol. 19, no. 2, p. 135, 2013.

[40] K. M. Mahmoud and A. S. Ammar, "Effect of N-acetylcysteine on cardiac injury and oxidative stress after abdominal aortic aneurysm repair: a randomized controlled trial," Acta Anaesthesiologica Scandinavica, vol. 55, no. 8, pp. 1015-1021, 2011. 


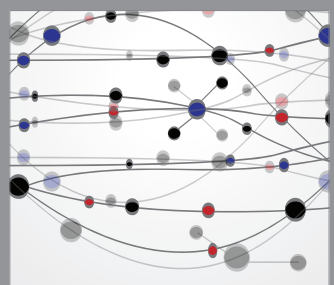

The Scientific World Journal
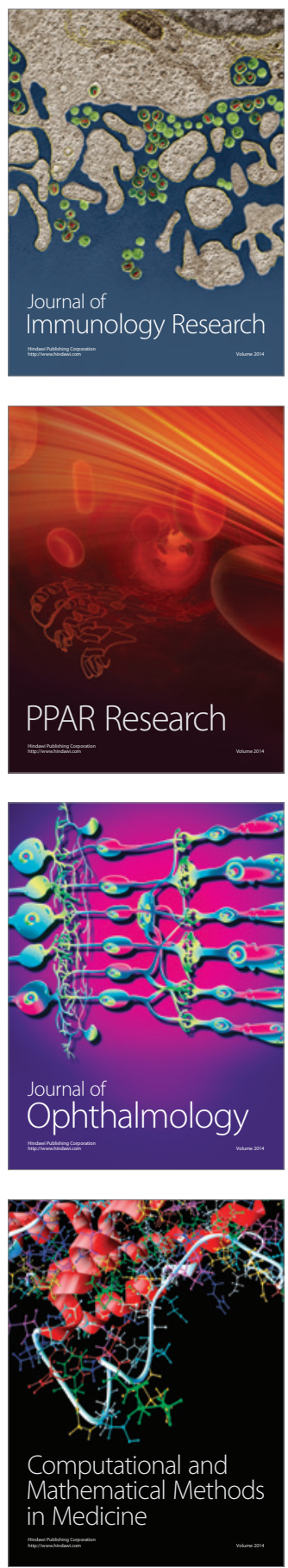

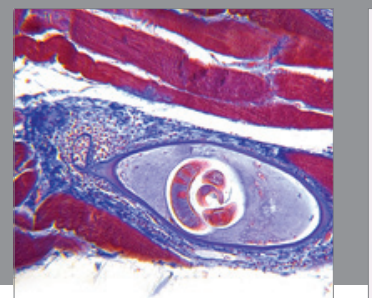

Gastroenterology

Research and Practice
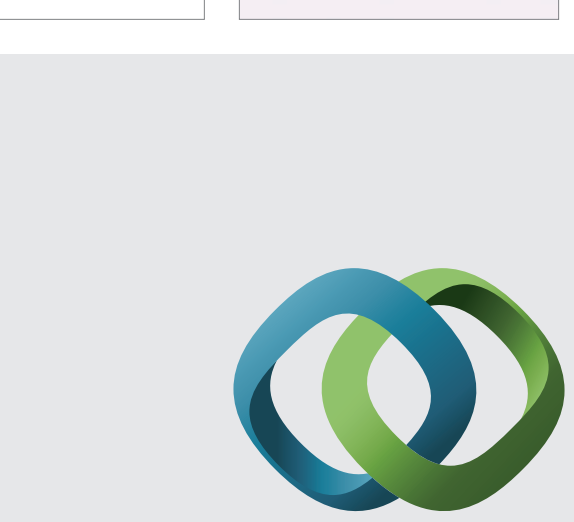

\section{Hindawi}

Submit your manuscripts at

http://www.hindawi.com
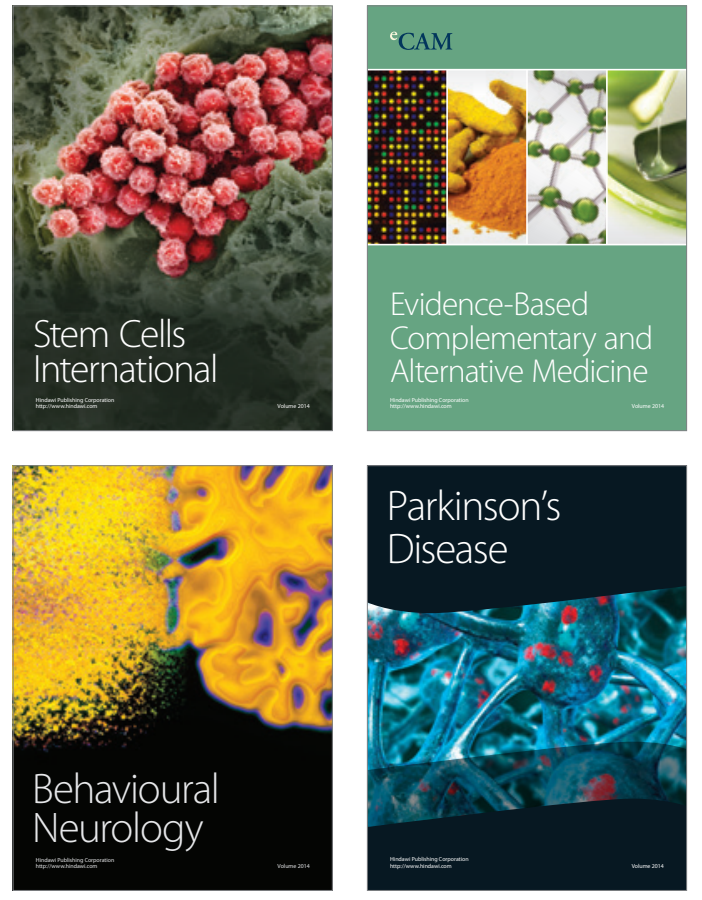
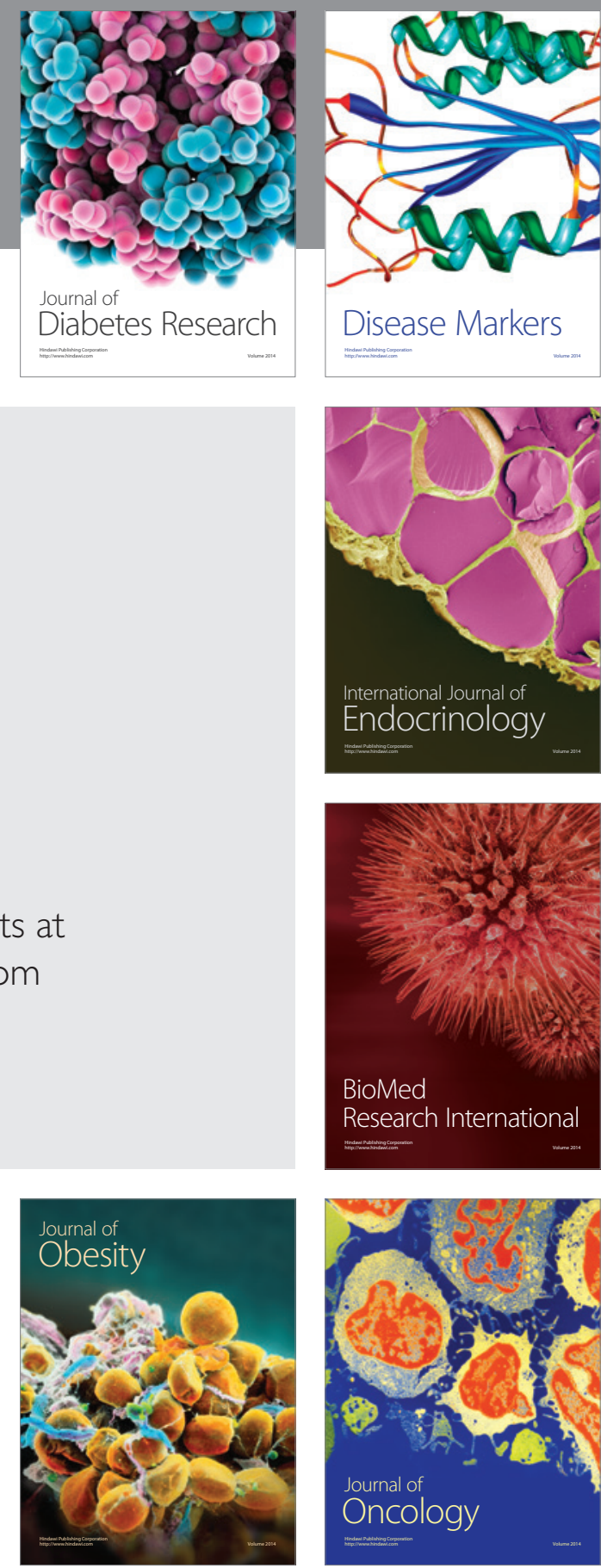

Disease Markers
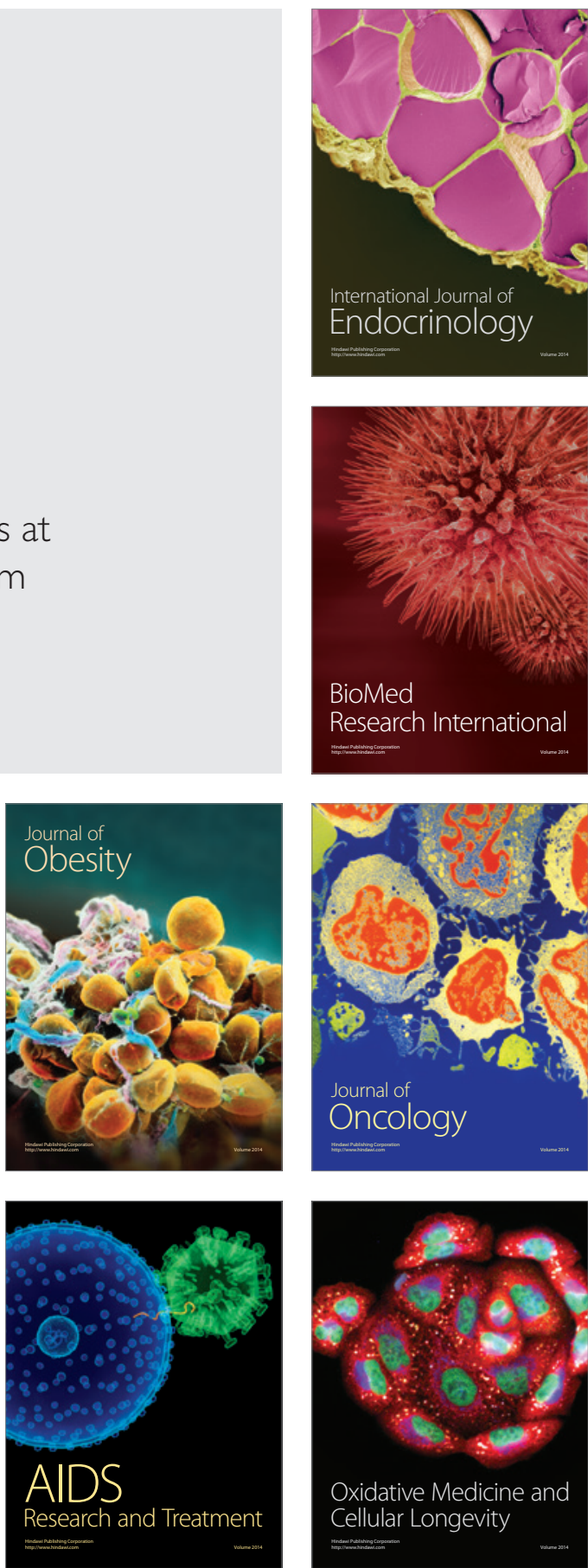